\title{
Serum antibody screening for non-human leukocyte antigen antibodies associated with antibody-mediate rejection reveals significance of anti-collagen type I and type III antibodies
} \author{
Eun Young Seong ${ }^{6}$, Hajeong Lee $^{3}$ \\ ${ }^{1}$ Division of Nephrology, Department of Internal Medicine, Korean Armed Forces Capital Hospital, Seongnam, Korea \\ ${ }^{2}$ Kidney Research Institute, Seoul National University Hospital, Seoul, Korea \\ ${ }^{3}$ Department of Internal Medicine, Seoul National University Hospital, Seoul, Korea \\ ${ }^{4}$ Division of Transplantation, Department of Surgery, Seoul National University Hospital, Seoul, Korea \\ ${ }^{5}$ Department of Pathology, Seoul National University Hospital, Seoul, Korea \\ ${ }^{6}$ Department of Laboratory Medicine, Seoul National University Hospital, Seoul, Korea
}

Sehoon Park ${ }^{1}$, Seung-Hee Yang ${ }^{2}$, Jiyeon $\mathrm{Kim}^{2}$, Semin $\mathrm{Cho}^{3}$, Sang-il $\mathrm{Min}^{4}$, Jongwon $\mathrm{Ha}^{4}$, Yon Su Kim ${ }^{3}$, Kyung Chul Moon ${ }^{5}$

Background: Additional study is warranted to determine the clinical significance of various non-human leukocyte antigen (HLA) antibodies on their association with the antibody-mediated rejection (ABMR) in kidney allografts.

Methods: The study included transplant recipients in one of the tertiary hospitals in Korea. We collected post-transplant sera from 68 ABMR, 67 T-cell mediated rejection (TCMR), and 83 controls without rejection cases and screened titers 39 non-HLA antibodies. We compared the non-HLA antibody titers among the study groups. We investigated their association with the risk of death-censored graft failure within the ABMR cases.

Results: The ABMR cases were diagnosed in later periods from transplantation and had a higher proportion of HLA-mismatched or HLA donor-specific antibody (DSA) positive cases when compared to the controls. Among the measured antibodies, anti-collagen type I $(P=0.001)$ and type III antibody $(P<0.001)$ titers were significantly higher in the ABMR cases when compared to the TCMR or no rejection controls. The both titers of anti-collagen type I (per 1 standard deviation [SD]; adjusted odds ratio [OR], 10.45; 95\% confidence interval [Cl], 2.52-66.62) and type III (per 1 SD; adjusted OR, 5.88; 95\% Cl, 1.84-28.98) antibody were significantly associated with presence of ABMR even after adjusting the presence of HLA-DSAs or other clinicopathologic findings. Within the ABMR group, a higher titer of anti-collagen type I (adjusted hazard ratio [HR], 1.82; 95\% Cl, 1.28-2.58) or type III (adjusted $\mathrm{HR}, 1.53 ; 95 \% \mathrm{Cl}, 1.14-2.07)$ antibody was associated with higher risk of death-censored graft failure.

Conclusions: Post-transplant anti-collagen I and collagen III antibodies may be novel non-HLA antibodies that are related to ABMR of kidney allografts.

Corresponding author: Sehoon Park

E-mail: mailofsehoon@gmail.com

(c) The Korean Society for Transplantation

This is an Open Access article distributed under the terms of the Creative Commons Attribution Non-Commercial License (http://creativecommons.org/licenses/by-nc/4.0/) which permits unrestricted non-commercial use, distribution, and reproduction in any medium, provided the original work is properly cited. 